\title{
CpG island shore methylation of ZFPM2 is identified in tetralogy of fallot samples
}

\author{
Wei Sheng ${ }^{1,2}$, Long Chen ${ }^{3}$, Huijun Wang ${ }^{1,2}$, Xiaojing Ma ${ }^{1,2}$, Duan Ma ${ }^{2,4}$ and Guoying Huang ${ }^{1,2}$
}

BACKGROUND: ZFPM2 gene plays an important role in heart morphogenesis and development of coronary vessels from epicardium, however, little is known regarding its epigenetic regulation in the pathogenesis of tetralogy of fallot (TOF).

METHODS: The methylation levels of ZFPM2 gene were measured by MassArray (Sequenom, San Diego, CA) and bisulfite sequencing polymerase chain reaction (PCR) (BSP). Real-time $P C R$ was performed to analyze the mRNA levels for ZFPM2 gene in the myocardium of TOF.

RESULTS: The methylation levels in the CpG island shore of ZFPM2 promoter were significantly higher in patients with TOF, with a median of $80.32 \%$ (interquartile range (IQR): $73.54-85.75 \%, N=42$ ), as compared to $59.63 \%$ in controls (IQR: 44.79-73.83\%; $P=0.0186, N=6$ ). No significant difference was observed in the methylation status at the $\mathrm{CpG}$ island of ZFPM2 promoter. The ZFPM2 mRNA levels were significantly lower in patients with TOF compared to that in the controls $(P$ $<0.05)$. The aberrant methylation values of ZFPM2 were negatively associated with significant changes in its mRNA level ( $r=$ $-0.40, P=0.008, N=42$ ).

CONCLUSION: Aberrant methylation status at the promoter CpG island shore of ZFPM2 gene may be associated with its gene transcription regulation in the TOF patients.

$\mathbf{T}$ etralogy of fallot (TOF) is a combination of four birth defects, including pulmonary stenosis, ventricular septal defect, overriding aortic roots, and hypertrophy of the right ventricle (1). TOF occurs in 3.6 out of every 10,000 live births and accounts for about $10 \%$ of all congenital heart defects (2). The mortality rate in untreated TOF patients reaches $50 \%$ by age $6 \mathrm{y}$; however, when receiving the cardiac surgery, children with simple forms of TOF enjoy good long-term survival with an excellent quality of life (3). Although treatment of TOF disease has advanced dramatically over the past few decades, the exact etiology remains unknown (4). Human genetic studies have identified numerous genes that are responsible for inherited and sporadic congenital heart diseases. Most of these genes, such as ZFPM2, encode transcription factors that regulate specific events in heart development, such as ventricular septum or outflow tract morphogenesis (5). ZFPM2 gene encodes a zinc finger protein and can physically interact with the N-terminal zinc finger of GATA4 to modulate its activity and thereby influence the transcription of target genes. This interaction is required for normal morphogenesis of heart during organogenesis (6). ZFPM2-/ mice embryo showed grossly normal in appearance at E12.5, however, at E13.5-15, the heart of ZFPM2 ${ }^{--}$mice exhibited the defects seen in human tetralogy of fallot and died at midgestation (7). The mutations in ZFPM2 have also been observed in the human TOF cases. De Luca et al. (8) have identified two missense variants in the ZFPM2 in the 1 of 178 patients with TOF. Although the whole exons of ZFPM2 were sequenced and several new mutations were observed in the TOF patients, the mutation percentage is very lower $(8-10)$. For the nongenetic mutation-caused TOF patients, the epigenetic mechanisms, such as DNA methylation, should be considered in the development of TOF $(11,12)$.

Currently, the DNA methylation is the most widely studied epigenetic modification in humans, which occurs almost exclusively in the context of $\mathrm{CpG}$ dinucleotides and regulates the transcriptional activity of genes by various mechanisms (13). The clusters of $\mathrm{CpG}$ dinucleotides consist of the $\mathrm{CpG}$ island regions and about $60 \%$ of gene promoters in human are overlapped with CpG islands and usually unmethylated in normal cells (14). The regions that lie in close proximity $(\sim 2 \mathrm{~kb})$ to the CpG islands are known as the CpG island shores (15). Although the CpG island shores have lower CpG site density, they frequently have functional $\mathrm{CpG}$ sites and show variable methylation levels and responsible for gene transcriptional activity. Most of the tissue-specific gene DNA methylation seems to occur, not at $\mathrm{CpG}$ islands, but at $\mathrm{CpG}$ island shores $(16,17)$.

Methylation within gene promoters has the closely functional relevance to gene expression regulation, and the aberrant methylation changes may contribute to many diseases (18). Although the mutations in ZFPM2 gene have been reported in patients with TOF, little is known about its DNA methylation changes in the TOF patients without gene mutations.

\footnotetext{
The first two authors and the last two authors contributed equally to this work.

${ }^{1}$ Children Hospital of Fudan University, Shanghai, China; ${ }^{2}$ Shanghai Key Laboratory of Prevention and Intervention of Birth Defects, Shanghai, China; ${ }^{3}$ Department of Forensic Medicine, Fudan University, Shanghai, China; ${ }^{4}$ Department of Biochemistry and Molecular Biology, Shanghai Medical College, Fudan University, Shanghai, China. Correspondence: Guoying Huang (gyhuang@shmu.edu.cn)

Received 5 March 2015; accepted 14 September 2015; advance online publication 30 March 2016. doi:10.1038/pr.2016.42
} 


\section{Articles | shengetal.}

The goal of the present study was to explore the DNA methylation changes of ZFPM2 and its epigenetic regulation in TOF patients. The potential results may provide important clues to assist the development of new treatments for TOF as well as give a deeper understanding of the etiology of this disease.

\section{RESULTS}

\section{Methylation Status Analyses for ZFPM2 Gene in TOF Patients and Controls}

The methylation status of ZFPM2 gene was analyzed using the Sequenom MassARRAY platform (Sequenom). We focused on the promoter region of ZFPM2 gene from $-2,000 \mathrm{bp}$ to $+200 \mathrm{bp}$ (relative to transcription start site). Based on the information of the ZFPM2 promoter region analyzed by the UCSC Genome Browser (Santa Cruz, San Francisco, CA), we designed the amplicons and polymerase chain reaction (PCR) primers using the EpiDesigner software (Sequenom) and found only one amplicon in the CpG island shore region (ZFPM2_ R1: -1,752bp $-1,323$ bp, Figure 1a), which is 430 base pairs in length and contains 6 CpG units. Primer sequences used are listed in Table 1.

The methylation level of ZFPM2_ R1 was measured in specimens from 10 TOF patients and 6 age-matched controls for the first screening. The results indicated that the methylation values for ZFPM2_R1 was significantly higher in the TOF patients, with a median of $83.00 \%$ (interquartile range (IQR): $78.50-85.42 \%, N=9$, one male, aged 19 mo was excluded), as compared to $59.63 \%$ in control subjects (IQR: $44.79-73.83 \%$; $P=0.0256, N=6$ ) (Figure 1b).
To explore the methylation status of promoter $\mathrm{CpG}$ island of ZFPM2 gene, which can not be detected by the Sequenom MassARRAY system (Sequenom), BSP was performed to measure the methylation level of ZFPM2_ R2 (-950bp -534bp, Figure 1a) located in the promoter $\mathrm{CpG}$ island of $Z F P M 2$ gene in two TOF patients and in two controls, which is 416 base pairs in length and contains $17 \mathrm{CpG}$ units. The BSP primers used in the study are listed in Table 1. As shown in Figure 1c, the methylation levels of the ZFPM2_R2 were very low and showed no significant difference between the TOF patients and controls.

\section{Validation of the Methylation Level of ZFPM2_R1 in TOF Patients and Controls}

According to the date of the first screening, the different methylation levels for ZFPM2_ R1 were further validated by the Sequenom MassARRAY system in a larger cohort of 33 TOF patients and six age-matched controls. As shown in the Figure 2a, the methylation value of ZFPM2_ R1 was significantly higher in patients with TOF, with a median value of 79.67\% (IQR: $68.33-86.30 \%, N=33$ ), while the control subjects are 59.63\% (IQR: $44.79-73.83 \% ; P=0.0279, N=6$ ).

Furthermore, when the ZFPM2_R1 methylation levels in the 33 TOF patients and the initial 9 TOF subjects were combined and compared to that of the control subjects, a significantly higher ZFPM2_R1 methylation level was found in TOF patients, with a median value of $80.32 \%$ (IQR: $73.54-85.75 \%$, $N=42$ ) as compared to $59.63 \%$ (IQR: $44.79-73.83 \%$; $P=$ $0.0186, N=6$, Figure $2 b$ ) in controls. a

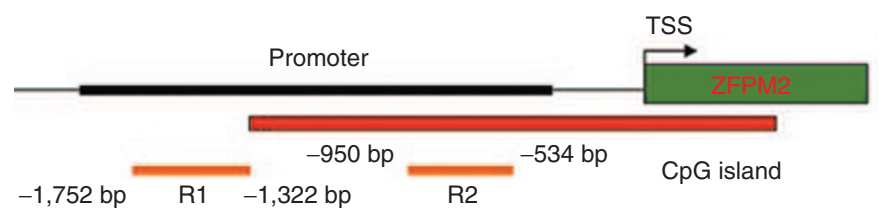

b

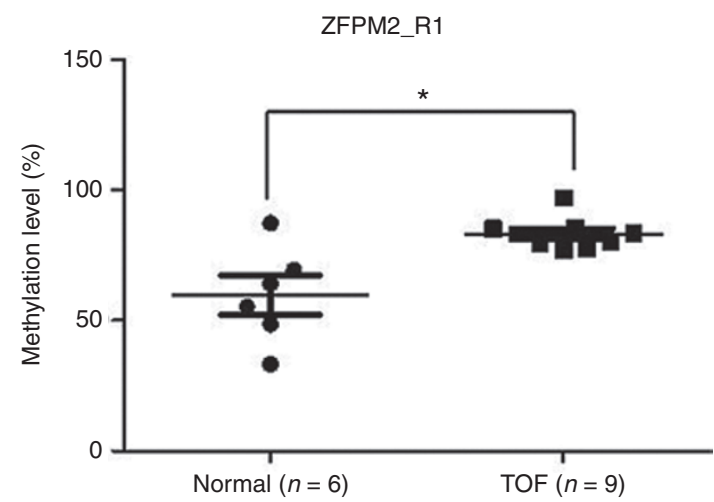

C N1

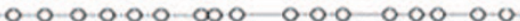
N1 $0.00000-0000000000$ $0.0000-000-0.00000-0.0$ $0.0-0.0000000000000$ 0.000000000000000 $0.0000-000-0.00000$ $0.0-0.0-000-0.0000-0.0$ $0.0-0.0-000-0.0-0.00-0.0$ $0.0 \cdot 0.0-000-0.00-00-0.0$ $00-0000000-000-000-00$ $-00000000000000-00$ N2 -0.000 - $000000-0.00-0.0$

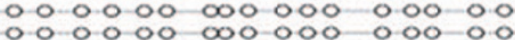

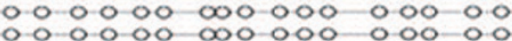

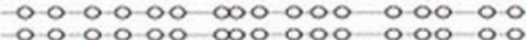
0.000000000000000 $0.0-0.00-\infty 00-00000000$ $0.000000000000-00$ 00000000000000000 T1 -00-000 00000000000 $00-0000-00000000000$ 000.0000000000000 00000000000000000 0000000000000000 00000000000000 $0000000000.000-00$ 00000000000.00000

T2 00000000000000000 $00-0000-000000000-00$ 00000000000000000 $00-00000000000000$ $-0000000000000000$ $-0000000000000000$ $-0000000000000000$ $-00-00000000000000$ $000000 \infty 000000000$

Figure 1. The methylation status for ZFPM2_R1 and ZFPM2_R2. (a) The schematic represents the distribution of ZFPM2_R1 and ZFPM2_R2 in the promoter region. (b) Median methylation levels for ZFPM2_R1 between normal and tetralogy of fallot subjects. (c) BSP sequencing results of ZFPM2_R2. All the values represent median with interquartile range. TSS, transcription start sites; red thick bars, CpG islands; R1, MassARRAY amplicon; R2, BSP sequencing region. ${ }^{*} P<0.05$ (Mann-Whitney test). BSP, bisulfite sequencing polymerase chain reaction. 
Table 1. Primer sets for MassArray methylation analysis and BSP

\begin{tabular}{lll}
\hline $\begin{array}{l}\text { Primers for MassArray } \\
\text { methylation analysis }\end{array}$ & Sequence & Position (bp) \\
\hline ZFPM2_R1 & Forward primer: GGGATTTATGTGAATTGTAGTGGAG ; reverse primer: AAAATTCTATAATCCCACCCTACCC & $-1,752 \sim-1,323$ \\
Primers for BSP:ZZFPM2_R1 & Forward primer: GGATTTATGTGAATTGTAGTGGA; reverse primer: AAAAAATTCTATAATCCCACCCT & $-1,751 \sim-1,319$ \\
ZFPM2_R2 & Forward primer: AGGAAGAGAGTTGTTTATTTTYGAAYGTGA; reverse primer:CTCAAACAAAATTAAAACATCTAA & $-950 \sim-534$ \\
\hline
\end{tabular}

a10-mer tag: cagtaatacgactcactatagggagaagg and ${ }^{\mathrm{T}} \mathrm{T} 7$ promoter tag: aggaagagag were added.

a

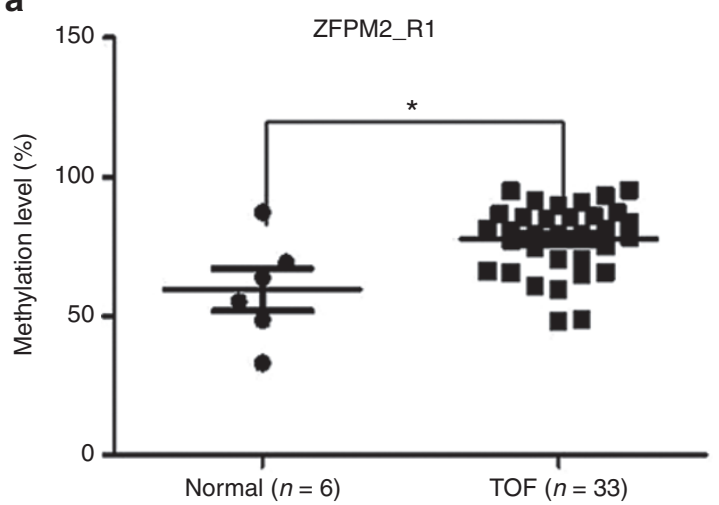

b

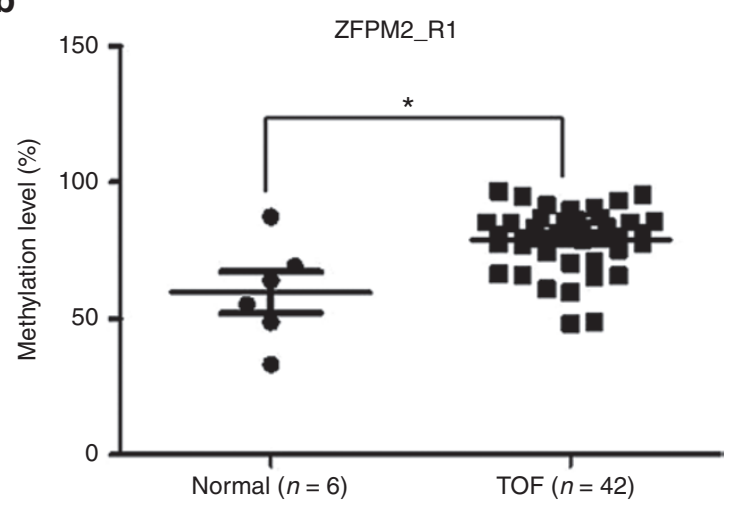

Figure 2. Median methylation levels for ZFPM2_R1 between normal controls and tetralogy of fallot patients. (a) In independent validated cohorts. (b) In combined group of screening and independent validated cohorts. ${ }^{*} P<0.05$ (Mann-Whitney test).

a

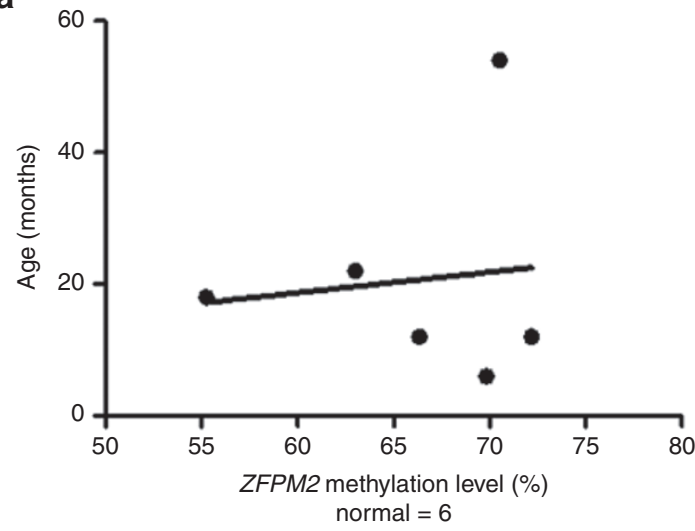

b

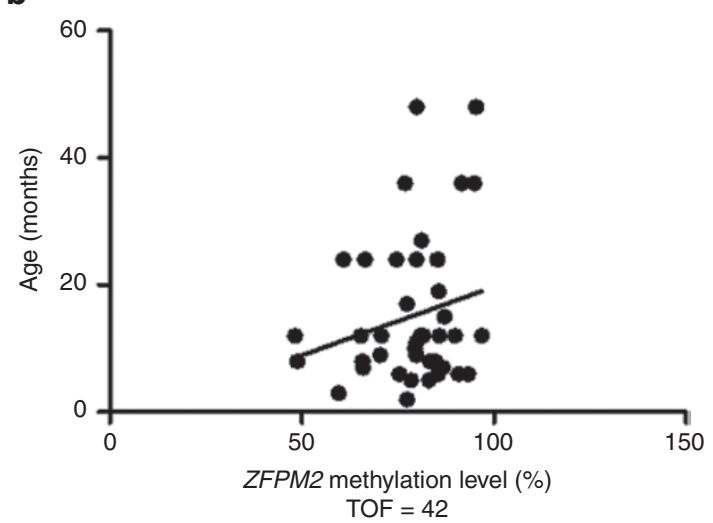

Figure 3. Correlation of ZFPM2 methylation level and age. (a) In the control group $(N=6)$. (b) In the tetralogy of fallot patients $(N=42)$.

Table 2. Primer sequences and product length for PT-PCR analysis

\begin{tabular}{llll}
\hline Genes & Forward primer $\left(5^{\prime} \rightarrow 3^{\prime}\right)$ & Reverse primer $\left(5^{\prime} \rightarrow 3^{\prime}\right)$ & Product length $(\mathrm{bp})$ \\
\hline ZFPM2 & AGGCTTCCTCAAATGGGTGTG & CCGAAGTCACGAAGTTGTG & 159 \\
B2M & TGCTGTCTCCATGTTTGATGTATCT & TCTCTGCTCCCCACCTCTAAGT & 161 \\
GAPDH & AGAAGGCTGGGGCTCATTTG & AGGGGCCATCCACAGTCTTC & 220 \\
\hline
\end{tabular}

Considering the difference of age in the individual subjects in the TOF patients and normal controls, which may influence the methylation values, a correlation of ZFPM2 methylation level with age was analyzed and no significant association was found between the ZFPM2 methylation values and age in the normal controls $(r=-0.174, P=0.714$; $N=6$, Figure $3 \mathrm{a})$ or in TOF patients $(r=0.115, P=0.467$; $N=42$, Figure $3 \mathbf{b}$ ).

\section{Expression Levels of ZFPM2 mRNA in Patients With TOF and Controls}

The mRNA expression level of the ZFPM2 gene was determined by quantitative real-time polymerase chain reaction in $42 \mathrm{TOF}$ patients and 6 controls. The primers used in this study are listed in Table 2.

The normalized $C_{t}$ mean $\left(\Delta C_{t}\right), \Delta \Delta C_{t}$, and relative quantification $\left(2^{-\triangle \Delta \mathrm{Ct}}\right)$ values for ZFPM2 gene in each samples are 
Table 3. The normalized $C_{t}$ mean $\left(\Delta C_{t}\right), \Delta \Delta C_{t^{\prime}}$ and $R Q\left(2^{-\Delta \Delta C t}\right)$ values for ZFPM2 gene in each individual samples

\begin{tabular}{|c|c|c|c|c|c|}
\hline \multirow[b]{2}{*}{ Sample ID } & \multirow{2}{*}{$\begin{array}{c}\text { Age } \\
\text { (months) }\end{array}$} & \multirow[b]{2}{*}{ Gender } & \multicolumn{3}{|c|}{ ZFPM2 mRNA levels } \\
\hline & & & $\Delta C_{\mathrm{t}}$ & $\Delta \Delta C_{t}$ & RQ value \\
\hline Control 1 & 6 & Male & 4.150335 & 0 & 1.000000 \\
\hline Control 2 & 12 & Female & 2.906015 & -1.24432 & 2.369072 \\
\hline Control 3 & 12 & Female & 2.143875 & -2.00646 & 4.017957 \\
\hline Control 4 & 18 & Male & 3.763645 & -0.38669 & 1.307386 \\
\hline Control 5 & 54 & Male & 3.823275 & -0.32706 & 1.254456 \\
\hline Control 6 & 22 & Male & 2.417975 & -1.73236 & 3.322712 \\
\hline TOF patient 1 & 8 & Female & 3.989945 & -0.16039 & 1.117590 \\
\hline TOF patient 2 & 12 & Female & 3.778595 & -0.37174 & 1.293913 \\
\hline TOF patient 3 & 6 & Male & 3.759325 & -0.39101 & 1.311311 \\
\hline TOF patient 4 & 6 & Male & 4.505353 & 0.355018 & 0.7818601 \\
\hline TOF patient 5 & 7 & Male & 3.932915 & -0.21742 & 1.162650 \\
\hline TOF patient 6 & 8 & Male & 3.338405 & -0.81193 & 1.755555 \\
\hline TOF patient 7 & 48 & Male & 4.060455 & -0.08988 & 1.064283 \\
\hline TOF patient 8 & 19 & Male & 3.432945 & -0.71739 & 1.644206 \\
\hline TOF patient 9 & 5 & Female & 4.419488 & 0.269153 & 0.8298066 \\
\hline TOF patient 10 & 19 & Male & - & - & - \\
\hline TOF patient 11 & 24 & Female & 4.177629 & 0.027294 & 0.9812594 \\
\hline TOF patient 12 & 24 & Female & 4.45089 & 0.300555 & 0.8119401 \\
\hline TOF patient 13 & 12 & Male & 3.671905 & -0.47843 & 1.393225 \\
\hline TOF pati & 3 & Male & 6.097789 & 1.947454 & 0.2592734 \\
\hline TOF patient 15 & 8 & Female & 3.690725 & -0.45961 & 1.375172 \\
\hline TOF patient 16 & 36 & Male & 4.168701 & 0.018366 & 0.9873501 \\
\hline TOF patient 17 & 7 & Female & 4.493055 & 0.34272 & 0.7885532 \\
\hline TOF patient 18 & 36 & Male & 3.504985 & -0.64535 & 1.564121 \\
\hline TOF patient 19 & 9 & Female & 4.54149 & 0.391155 & 0.762519 \\
\hline TOF patient 20 & 5 & Male & 4.072995 & -0.07734 & 1.055074 \\
\hline TOF & 7 & Male & 4.06 & -0.08466 & 1.060437 \\
\hline TOF & 12 & le & 3.1 & -0.98915 & 1.985009 \\
\hline TOF patient 23 & 12 & Male & 4.153387 & 0.003052 & 0.9978868 \\
\hline TOF patient 24 & 7 & Male & 4.190932 & 0.040597 & 0.9722528 \\
\hline TOF patient 25 & 8 & Male & 5.771464 & 1.621129 & 0.325081 \\
\hline TOF patient 26 & 24 & Male & 4.338642 & 0.188307 & 0.8776352 \\
\hline TOF patient 27 & 6 & Male & 4.351883 & 0.201548 & 0.869617 \\
\hline TOF patient 28 & 10 & Male & 3.763545 & -0.38679 & 1.307479 \\
\hline TOF patient 29 & 8 & Male & 4.236418 & 0.086083 & 0.9420774 \\
\hline TOF patient 30 & 8 & Fem & 3.123035 & -1.0273 & 2.038205 \\
\hline TOF patient 31 & 11 & Female & 3.877495 & -0.27284 & 1.208180 \\
\hline TOF patient 32 & 5 & Male & 3.652785 & -0.49755 & 1.411811 \\
\hline TOF patient 33 & 48 & Female & 4.032105 & -0.11823 & 1.085406 \\
\hline TOF patient 34 & 24 & Male & 3.888285 & -0.26205 & 1.199182 \\
\hline TOF patient 35 & 36 & Female & 4.394542 & 0.244207 & 0.8442799 \\
\hline TOF patient 36 & 12 & Male & 4.544683 & 0.394348 & 0.7608329 \\
\hline TOF patient 37 & 12 & Female & 3.716115 & -0.43422 & 1.351179 \\
\hline TOF patient 38 & 6 & Male & 3.598315 & -0.55202 & 1.466137 \\
\hline TOF patient 39 & 9 & Male & 4.147765 & -0.00257 & 1.001780 \\
\hline TOF patient 40 & 24 & Female & 3.858305 & -0.29203 & 1.224361 \\
\hline TOF patient 41 & 12 & Male & 4.082775 & -0.06756 & 1.047943 \\
\hline TOF patient 42 & 48 & Female & 4.706603 & 0.556268 & 0.6800593 \\
\hline TOF patient 43 & 12 & Male & 4.404327 & 0.253992 & 0.8385729 \\
\hline
\end{tabular}

$R Q$, relative quantification; $T O F$, tetralogy of fallot. listed in Table 3. The relative quantification value $\left(2^{-\Delta \Delta C t}\right)$ of control 1 was set as 1 and used for normalizations for all samples. As shown in Table 4, ZFPM2 mRNA relative expression levels (relative quantification values) showed significantly lower in patients with TOF compared with controls $(P<0.05)$.

\section{Association Between the Methylation Statuses and mRNA Level of ZFPM2 Gene}

A correlation analysis was performed to identify any relationships between ZFPM2 methylation statuses and its respective mRNA levels in TOF patients.

As shown in Figure 4a, a significant association between the methylation statuses of ZFPM2_R1 and its mRNA levels was observed for the nine TOF patients in the first screening $(r=-0.75, P=0.026, N=9)$. In a larger cohort of 33 TOF patients, there was a strong association between the methylation statuses and mRNA level for the ZFPM2 gene $(r=-0.42, P=0.014, N=$ 33 , Figure $4 \mathrm{~b})$. Interestingly, a higher significant correlation was found between the ZFPM2_R1 methylation statuses and mRNA levels ( $r=-0.40, P=0.008, N=42$, Figure $4 \mathrm{c})$ in the combination cohort of the initial 9 and independent 33 TOF patients.

To further explore whether DNA methylation regulates gene expression of ZFPM2 in vitro, we used human cardiac myocyte (HCM) cell lines (ScienCell, San Diego, CA) in this study due to lack of well-established TOF disease cell lines. The HCM cell lines were treated with a DNA methyltransferase inhibitor (5-aza-2'-deoxyctidie) (Sigma, St.Louis, MO), which can inhibit the activity of DNA methyltransferase and reduce the DNA methylation level of genome. We then analyzed the methylation status and mRNA levels of ZFPM2 gene before and after treatment with 5-aza-2'-deoxyctidie in this cell. As shown in Figure 5a,b, the methylation values of the ZFPM2_ R1 were very low and showed a significant difference before and after treatment with 5-aza-2'-deoxyctidie. The BSP primers for ZFPM2_ R1 used in the study are listed in Table 1. Moreover, we found a significantly increased expression of the ZFPM2 gene in the cell treated with 5-aza2 '-deoxyctidine Figure $5 \mathrm{c}$. These data indicated that the promoter methylation of ZFPM2 gene maybe associated with its gene expression.

\section{DISCUSSION}

Epigenetic refers to the study of heritable changes in gene regulation occurring without changes in the DNA sequence, including DNA methylation, histone modification, chromatin remodeling, and long noncoding RNAs. Epigenetic plays a key role in the regulation of tissue homeostasis and disease development (19). The development of TOF disease may be associated with the abnormality of epigenetic regulation. In our previous studies, we have observed that LINE-1 methylation levels, which may serve as a potential indicator of global DNA methylation status, were lower in the cardiac tissue of TOF patients and increased the risk of TOF development (20). The decreased LINE- 1 methylation levels may be caused by the lower expression of DNMT1 and DNMT3B (21). In the present study, we focus on the DNA methylation changes within the 
promoter region of the ZFPM2 gene. We used the Sequenom MassARRAY platform (Sequenom) to analyze the methylation status of ZFPM2 gene. The accuracy of this approach for quantifying methylated and unmethylated DNA has been validated by the Sequenom group (22) and our previous study (23). The methylation levels for the promoter region of ZFPM2 gene were initially explored in the cardiac tissues of 10 TOF patients and 6 age-matched controls, and a significant difference in the methylation levels of CpG island shore of ZFPM2 gene $\left(Z F P M 2 \_R 1\right)$ was observed $(P=0.0256)$. Moreover, BSP was used to explore the methylation change in the promoter CpG island of ZFPM2 gene (ZFPM2_R2) and no significant difference was observed (Figure 1c).

Table 4. Relative mRNA expression levels of ZFPM2 in TOF patients and controls

\begin{tabular}{lccc}
\hline Gene & $\begin{array}{c}\text { Control (RQ value }{ }^{\mathrm{a}}: \\
\text { mean } \pm \text { SD, } N=6)\end{array}$ & $\begin{array}{c}\text { TOF (RQ value: } \\
\text { mean } \pm \text { SD, } N=42)\end{array}$ & $P_{\text {value }}{ }^{\mathrm{b}}$ \\
\hline ZFPM2 & $2.21 \pm 1.24$ & $1.11 \pm 0.37$ & $<0.0001$
\end{tabular}

aThe RQ values ( $2^{-\Delta C t}$ ) for control 1 was set as 1 and used for normalization for all samples; bunpaired $t$-test was performed.

$R Q$, relative quantification; TOF, tetralogy of fallot
ZFPM2 is a multi-zinc-finger protein that is coexpressed with Gata4 in the developing heart beginning at E8.5. Svensson et al. (24) used targeted mutagenesis to explore the role of $Z f p m 2$ in normal cardiac development and found Zfpm2-deficient mice died of congestive heart failure at E13 with a syndrome of tricuspid atresia that includes an elongated left ventricular outflow tract, rightward displacement of the aortic valve, and pulmonic stenosis. Moreover, miR-130a has also been reported to regulate the ZFPM2 gene expression and plays an important role in the regulation of cardiac development (25). Promoter DNA methylation of a gene is tightly associated with its transcription activity. DNA hyper-methylation of $\mathrm{CpG}$ islands in the gene promoter region has been widely reported to associate with the gene silencing (26). In the current study, we examined the methylation status of $\mathrm{CpG}$ islands in the promoter area and mRNA levels of ZFPM2 gene. Although the ZFPM2 mRNA levels were significantly lower in patients with TOF disease compared with controls $(P<0.05)$, the methylation status of promoter $\mathrm{CpG}$ island was very low and there was no significant difference between the TOF patients and controls. These findings suggested that the methylation levels of CpG islands in the ZFPM2 promoter may not influence its gene expression. a

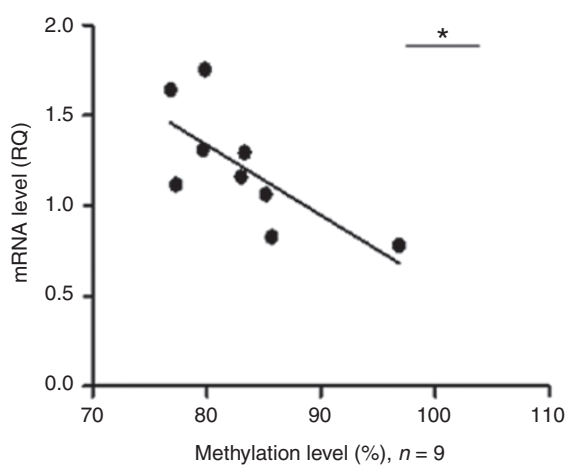

b

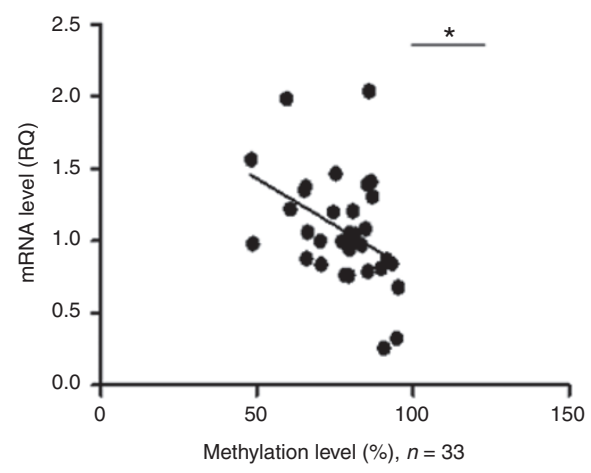

c

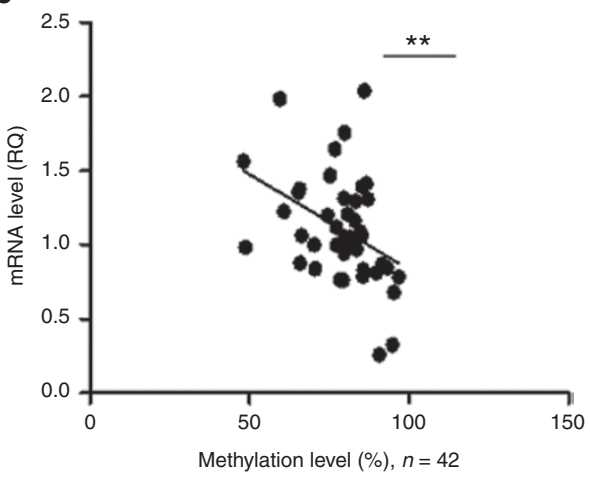

Figure 4. Association of ZFPM2 methylation status with its mRNA levels in tetralogy of fallot patients. (a) In the first screening cohort; (b) in independent validated cohorts; (c) in combined group of first screening and independent validated cohorts. ${ }^{*} P<0.05,{ }^{* *} P<0.01$.

a

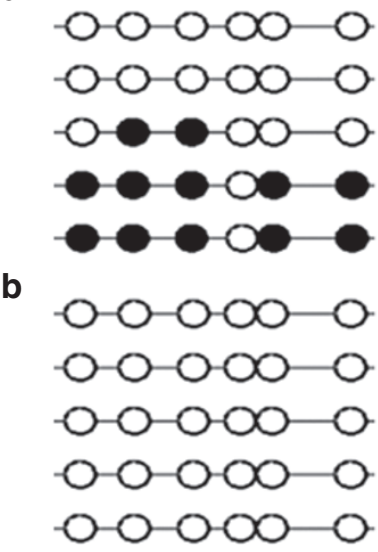

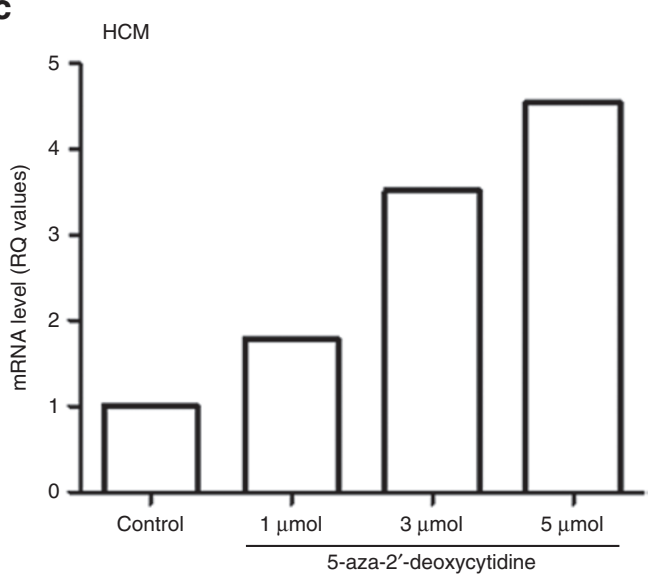

Figure 5. BSP sequencing results of ZFPM2_R1 and mRNA expression levels of ZFPM2. (a) BSP sequencing results of ZFPM2_R1 in HCM cells; (b) BSP sequencing results of ZFPM2_R1 in HCM cells treated with 5-aza-2'-deoxycytidine at $5 \mu \mathrm{mol}$ for $48 \mathrm{~h}$; (c) mRNA expression levels of ZFPM2 in the HCM cell before and after treated with 5-aza-2'-deoxycytidine at 1, 3, and $5 \mu \mathrm{mol}$, respectively, for $48 \mathrm{~h}$. 
Table 5. Anagraphical characteristics of TOF patients and control subjects

\begin{tabular}{|c|c|c|c|}
\hline \multirow[b]{2}{*}{ Characteristic } & \multicolumn{2}{|c|}{ TOF $(N=43)$ (months) } & \multirow[b]{2}{*}{$\begin{array}{c}\text { Control }(N=6) \\
\text { (months) }\end{array}$} \\
\hline & $\begin{array}{c}\text { First screening } \\
\text { cohort } \\
(N=10)\end{array}$ & $\begin{array}{l}\text { Independent } \\
\text { validated cohort } \\
\quad(N=33)\end{array}$ & \\
\hline \multicolumn{4}{|c|}{ Age (median $\left.\left(\mathrm{IQR}^{*}\right)\right)$} \\
\hline Gender & $8.0(6.0-19.0)$ & $12.0(7.5-24.0)$ & $15.0(10.5-30.0)$ \\
\hline Male (\%) & $7(70.0)$ & $20(60.6)$ & $4(66.7)$ \\
\hline Female (\%) & $3(30.0)$ & $13(39.4)$ & $2(33.3)$ \\
\hline
\end{tabular}

The CpG island shore refers to the region located in close proximity ( $2 \mathrm{~kb})$ to the $\mathrm{CpG}$ islands. Recent study has proved that $76 \%$ of differential tissue methylation regions were not located in CpG islands, but in the $\mathrm{CpG}$ island shores (27). Rao et al. (28) have reported that, within the promoter of Cavl gene, the $\mathrm{CpG}$ island shore methylation, not the $\mathrm{CpG}$ islands, regulates the gene expression in the breast cancer. Although there is no significant difference in the $\mathrm{CpG}$ island methylation of $Z F P M 2$ gene in the initial study, the $\mathrm{CpG}$ island shore methylation in the ZFPM2 promoter showed significant increased in the TOF patients (Figure $1 \mathbf{l b}$ ). The differential methylation levels of the $\mathrm{CpG}$ island shore were then validated and confirmed in an independent cohort (Figure 2a). When combining the data of the two independent cohorts, interestingly, the methylation levels of the $\mathrm{CpG}$ island shore were significant differently even more between the TOF patients and controls (Figure 2b).

Considering the age difference in the individual subjects, which have been reported to influence DNA methylation patterns (29), we analyzed the association of ZFPM2 CpG island shore methylation with age and found no significant association in the normal controls (Figure 3a) or in TOF patients (Figure $3 b$ ), suggesting that the age may not influence the ZFPM2 methylation levels.

Moreover, we also analyzed the association of methylation level of ZFPM2 gene with gene expression in the patients with TOF disease. A negative association between methylation status and mRNA level for ZFPM2 gene was found in the TOF cases (Figure 4). To further explore whether the DNA methylation regulates the gene expression of ZFPM2 in vitro, we used HCM cell lines in this study due to lack of well-established TOF disease cell lines. The HCM cell lines were treated with a DNA methyltransferase inhibitor (5-aza-2'-deoxyctidie), which can inhibit the activity of DNA methyltransferase and reduce the DNA methylation level of genome. Interestingly, we observed a reduced DNA methylation value and increased expression for ZFPM2 gene in cells after treatment with 5-aza2 -deoxyctidie. These findings indicated that promoter methylation of the ZFPM2 gene may influence the gene expression and contribute to the development of TOF disease.

As it is difficult to collect cardiac tissue samples from healthy control and TOF patients, we were unable to obtain enough complete matched samples. Further studies with larger samples or in cell level with more methods are warranted to confirm our findings and reveal how aberrant methylation change contributes to TOF development.

\section{Conclusion}

The present study has explored the methylation status of ZFPM2 and found significant changes of methylation levels at the CpG island shore in the ZFPM2 promoter region. Moreover, aberrant methylation status of ZFPM2 genes showed significant negative correlations with its corresponding mRNA expressions, indicating that the DNA methylation changes may contribute to their transcription regulation in the TOF patients.

\section{METHODS}

\section{Patients and Controls}

All surgical specimens of TOF subjects were obtained from the Children's Hospital of the Fudan University, Shanghai, China. Cardiovascular diagnosis was performed by echocardiography and confirmed by surgery. The surgical procedure is same for all despite different ages. Moreover, we mainly focused on the methylation changes in the TOF subjects, while the patients with other problem and defects were excluded in this study, which included heart failure, trisomy 21, 22q11 deletion, gene mutation, other chromosomal anomalies, or extracardiac major- or minor-associated anomalies.

A total of 43 patients with TOF were recruited in this study. Ten patients with TOF in first screening cohort included 7 (70\%) males and $3(30 \%)$ females, ranging in age from 5.0 to $48.0 \mathrm{mo}(8.0$ (6.019.0), median (interquartile range)). Thirty-three patients with TOF in the independent validated cohort included $20(60.6 \%)$ males and 13 (39.4\%) females, ranging in age from 3.0 to $48.0 \mathrm{mo}(12.0$ (7.5-24.0)).

The control group was taken from autopsy specimens from healthy subjects. The control subjects were collected at the forensic medicine department of the Fudan University, Shanghai, China. The postmortem interval for the control subjects was as shortly as possible, not more than $24 \mathrm{~h}$. Six age-matched control subjects were collected for the study, including four (66.7\%) males and two (33.3\%) females, ranging in age from 6.0 to $54.0 \mathrm{mo}(15.0(10.5-30.0))$. Anagraphical characteristics of the study subjects were summarized in Table 5 .

All the tissue samples were obtained from right ventricular outflow tract and saved in RNA later solution (Ambion, Austin, TX) immediately after surgical resection or autopsy and stored until use. So, the cellular composition or tissue quantity of controls is comparable to that of TOF patients, excluding any tissue heterogeneity that may affect methylation results.

Written informed consents were obtained from the parents or relatives of all the study subjects. This study was approved by the local ethics committee of the Fudan University.

\section{DNA Extraction and Sodium Bisulfite Conversion}

A QIA amp DNA Mini Kit (Qiagen, Hilden, Germany) was used to extract genomic DNA from the heart tissue samples of TOF and normal control subjects according to manufacturer's instructions. The concentration and purity of genomic DNA were determined by absorbance at 260 and $280 \mathrm{~nm}$ by NanoDrop 1000 Spectrophotometer (Thermo Scientific, Wilmington). EZ DNA Methylation Kit (Zymo Research, Orange, CA) was used to perform sodium bisulfite modification for the genomic DNA strictly according to manufacturer's instructions. The bisulfite converted genomic DNA was resuspended in $10 \mu \mathrm{l}$ elution buffer and stored at $-80{ }^{\circ} \mathrm{C}$ until the samples were ready for analysis.

\section{Quantitative MassARRAY Analysis of Gene Methylation Status}

Quantitative methylation analysis for ZFPM2 gene was performed using the EpiTyper by MassArray (Sequenom) based upon base-specific cleavage and MALDI-TOF mass spectrometry, as recommended by the manufacturer. The robustness of this approach for quantifying 


\section{The epigenetic regulation of ZFPM2 gene}

methylated and unmethylated DNA has been demonstrated by the Sequenom groups (22). The promoter regions of the ZFPM2 gene were analyzed by the UCSC Genome Browser (Santa Cruz). The amplicons and PCR primers used in this system were designed with the EpiDesigner software (Sequenom). For each reverse primer, an additional T7 promoter tag for in vivo transcription was added, as was a 10-mer tag on the forward primer to adjust for the melting temperature differences. All experiments were performed as we described before (20). The methylation status for ZFPM2 CpG island was detected by bisulfite sequencing PCR (BSP) (30).

\section{RNA Extraction and Quantitative real-time PCR}

Total RNA was extracted from all tested heart tissue samples using Trizol Reagent (Invitrogen, Carlsbad, CA) according to the manufacturer's instructions. The extracted RNA quality and integrity were validated before use. RNA was reverse-transcribed using PrimeScript RT reagent Kit with gDNA Eraser (Perfect Real Time) (Takara Biotechnology, Dalian, China) and integrity of synthesized cDNA was confirmed using glyceraldehyde 3-phosphate dehydrogenase $(\mathrm{GAPDH})$ as endogenous control. Quantitative real-time PCR was performed using SYBR Premix Ex Taq GC (Perfect Real Time, TaKaRa). The reaction volume includes $5 \mu$ l SYBR Premix Ex Taq GC, $0.2 \mu \mathrm{mol} / \mathrm{l}$ of each primer, $0.2 \mu \mathrm{l}$ ROX1 Reference Dye and $100 \mathrm{ng}$ cDNA. Reactions were performed in triplicate and analyzed using an ABI 7900 Sequence Detection System (Applied Biosystems, Foster, CA). The standard $2^{-\Delta \Delta C t}$ method was used to calculate the relative expression levels of mRNA. The $\beta-2$ microglobulin $(B 2 M)$ and the GAPDH gene were used as the endogenous controls for normalization in this study.

\section{Cell Culture and In Vitro Methylation Status and mRNA Levels of ZFPM2 Gene}

HCM cell lines were purchased from ScienCell Research Laboratories (ScienCell) and used in this study due to lack of well-established TOF disease cell lines. Cells were cultured in Dulbecco's Minimum Essential Medium supplemented with $10 \%$ fetal bovine serum (Gibco, $\mathrm{NY}$ ) and penicillin-streptomycin at the condition of $37^{\circ} \mathrm{C}$ in $5 \% \mathrm{CO}_{2}$. The HCM cells were treated with 5-aza-2'-deoxycytidine (Sigma) at $1^{\circ}$, 3 , and $5 \mu \mathrm{mol}$ respectively for $48 \mathrm{~h}$. Medium was changed daily with fresh drugs. The genomic DNA and RNA of the cell before and after treatment with 5-aza-2'-deoxyctidie (Sigma) were extracted for the analysis of methylation status and mRNA levels. The methylation status of ZFPM2_R1 was determined by BSP and mRNA level was measured by real-time RT-PCR assay as described above.

\section{Statistical Analysis}

Data were analyzed using GraphPad Prism (version 5.0; GraphPad Software, San Diego, CA). Mann-Whitney $U$-test or unpaired t test was performed to evaluate the significance of any differences between TOF and control groups. Spearman correlation analysis was performed to evaluate the correlations between the methylation status of ZFPM2 gene and its mRNA levels. All statistical analysis was twosided and $P<0.05$ was considered statistically significant.

\section{STATEMENT OF FINANCIAL SUPPORT}

This work was supported by grants from the National Natural Sciences Foundation of China (81570282, 81570283, and 81370198), Shanghai Municipal Commission of Science and Technology Research Project (11JC1401400).

Disclosure: The authors declare that they have no competing interests.

\section{REFERENCES}

1. Ho S, McCarthy KP, Josen M, Rigby ML. Anatomic-echocardiographic correlates: an introduction to normal and congenitally malformed hearts. Heart 2001;86 Suppl 2:II3-11.

2. Bédard E, McCarthy KP, Dimopoulos K, Giannakoulas G, Gatzoulis MA, Ho SY. Structural abnormalities of the pulmonary trunk in tetralogy of Fallot and potential clinical implications: a morphological study. J Am Coll Cardiol 2009;54:1883-90.

3. Starr JP. Tetralogy of fallot: yesterday and today. World J Surg 2010;34: 658-68.
4. Di Felice V, Zummo G. Tetralogy of fallot as a model to study cardiac progenitor cell migration and differentiation during heart development. Trends Cardiovasc Med 2009;19:130-5.

5. Bruneau BG. The developmental genetics of congenital heart disease. Nature 2008;451:943-8.

6. Hirai M, Ono K, Morimoto T, et al. FOG-2 competes with GATA-4 for transcriptional coactivator $\mathrm{p} 300$ and represses hypertrophic responses in cardiac myocytes. J Biol Chem 2004;279:37640-50.

7. Tevosian SG, Deconinck AE, Tanaka M, et al. FOG-2, a cofactor for GATA transcription factors, is essential for heart morphogenesis and development of coronary vessels from epicardium. Cell 2000;101:729-39.

8. De Luca A, Sarkozy A, Ferese R, et al. New mutations in ZFPM2/FOG2 gene in tetralogy of Fallot and double outlet right ventricle. Clin Genet 2011;80:184-90.

9. Tan ZP, Huang C, Xu ZB, Yang JF, Yang YF. Novel ZFPM2/FOG2 variants in patients with double outlet right ventricle. Clin Genet 2012;82:466-71.

10. Huang $X$, Niu W, Zhang Z, et al. Identification of novel significant variants of ZFPM2/FOG2 in non-syndromic Tetralogy of Fallot and double outlet right ventricle in a Chinese Han population. Mol Biol Rep 2014;41:2671-7.

11. Pierpont ME, Basson CT, Benson DW Jr, et al.; American Heart Association Congenital Cardiac Defects Committee, Council on Cardiovascular Disease in the Young. Genetic basis for congenital heart defects: current knowledge: a scientific statement from the American Heart Association Congenital Cardiac Defects Committee, Council on Cardiovascular Disease in the Young: endorsed by the American Academy of Pediatrics. Circulation 2007;115:3015-38.

12. Liu L, Li Y, Tollefsbol TO. Gene-environment interactions and epigenetic basis of human diseases. Curr Issues Mol Biol 2008;10:25-36.

13. Goll MG, Bestor TH. Eukaryotic cytosine methyltransferases. Annu Rev Biochem 2005;74:481-514.

14. Vanderkraats ND, Hiken JF, Decker KF, Edwards JR. Discovering highresolution patterns of differential DNA methylation that correlate with gene expression changes. Nucleic Acids Res 2013;41:6816-27.

15. Irizarry RA, Ladd-Acosta $C$, Wen $B$, et al. The human colon cancer methylome shows similar hypo- and hypermethylation at conserved tissuespecific CpG island shores. Nat Genet 2009;41:178-86.

16. Doi A, Park IH, Wen B, et al. Differential methylation of tissue- and cancerspecific CpG island shores distinguishes human induced pluripotent stem cells, embryonic stem cells and fibroblasts. Nat Genet 2009;41:1350-3.

17. Baccarelli A, Rienstra M, Benjamin EJ. Cardiovascular epigenetics: basic concepts and results from animal and human studies. Circ Cardiovasc Genet 2010;3:567-73.

18. Portela A, Esteller M. Epigenetic modifications and human disease. Nat Biotechnol 2010;28:1057-68.

19. Ohtani K, Dimmeler S. Epigenetic regulation of cardiovascular differentiation. Cardiovasc Res 2011;90:404-12.

20. Sheng W, Wang H, Ma X, et al. LINE-1 methylation status and its association with tetralogy of fallot in infants. BMC Med Genomics 2012; $5: 20$.

21. Sheng W, Qian Y, Wang H, et al. Association between mRNA levels of DNMT1, DNMT3A, DNMT3B, MBD2 and LINE-1 methylation status in infants with tetralogy of Fallot. Int J Mol Med 2013;32:694-702.

22. Ehrich M, Turner J, Gibbs P, et al. Cytosine methylation profiling of cancer cell lines. Proc Natl Acad Sci USA 2008;105:4844-9.

23. Sheng W, Qian Y, Wang H, et al. DNA methylation status of NKX25, GATA4 and HAND1 in patients with tetralogy of fallot. BMC Med Genomics 2013;6:46.

24. Svensson EC, Huggins GS, Lin H, et al. A syndrome of tricuspid atresia in mice with a targeted mutation of the gene encoding Fog-2. Nat Genet 2000;25:353-6.

25. Kim GH, Samant SA, Earley JU, Svensson EC. Translational control of FOG-2 expression in cardiomyocytes by microRNA-130a. PLoS One 2009;4:e6161.

26. Weisenberger DJ, Siegmund KD, Campan M, et al. CpG island methylator phenotype underlies sporadic microsatellite instability and is tightly associated with BRAF mutation in colorectal cancer. Nat Genet 2006;38: 787-93. 


\section{Articles | Sheng et al.}

27. Yuferov V, Nielsen DA, Levran O, et al. Tissue-specific DNA methylation of the human prodynorphin gene in post-mortem brain tissues and PBMCs. Pharmacogenet Genomics 2011;21:185-96.

28. Rao X, Evans J, Chae H, et al. CpG island shore methylation regulates caveolin-1 expression in breast cancer. Oncogene 2013;32:4519-28.
29. Eden A, Gaudet F, Waghmare A, Jaenisch R. Chromosomal instability and tumors promoted by DNA hypomethylation. Science 2003;300:455.

30. Reed K, Poulin ML, Yan L, Parissenti AM. Comparison of bisulfite sequencing PCR with pyrosequencing for measuring differences in DNA methylation. Anal Biochem 2010;397:96-106. 